\title{
Osteoimmunology: An Evolving Discipline
}

\section{Santos Castañeda ${ }^{1 *}$ and Luis Arboleya ${ }^{2}$}

${ }^{1}$ Servicio de Reumatología, Hospital Universitario de La Princesa, C/ Diego de León 62, 28006 Madrid. IIS-Princesa, Universidad Autónoma, Madrid, Spain ${ }^{2}$ Sección de Reumatología, Hospital Universitario Central de Asturias, C/ Celestino Villamil s/n, 33006 Oviedo, Spain

Osteoimmunology is an emerging interdisciplinary field that focuses on the cellular and molecular events underlying the interplay between bone and immune system. The term "osteoimmunology" was first employed in 2000 to describe the interactions between cells from the immune and skeletal systems [1]. The discovery of the receptor activator NF- $\mathrm{BB}$ (RANK) ligand (RANKL), the RANKL/RANK/ osteoprotegerin (OPG) pathway and the identification of their pivotal role in osteoclast activation, have been crucial in the development of this discipline $[2,3]$.

Given that immune cells and haematopoietic cells originate in the bonemarrow, it is perhaps not surprising that there is a cross-talkbetween these two systems [4-6]. Some of the molecular pathways involved in bone remodelling are also involved in regulating hematopoiesis. Moreover, multiple cytokines from lymphocytes, macrophages and Dendritic Cells (DC), influence bone remodelling and there are actually several regulatory molecules common to osteoclasts and immune cells, such as cytokines, chemokines, receptors, signalling molecules and transcription factors, all of which influence each other mutually [4]. Thus, the activity of the immune and skeletal systems should now be reconsidered in the more holistic context of the autoimmune system. In recent years, our understanding of the relationships between the cells and molecules that act in the osteoimmune system has advanced, not only those at play in normal physiological conditions but also, in diseases as far as arthritis, osteoporosis, periodontal disease or cancer. In fact, by studying the interaction of osteoclasts and T lymphocytes, we now better understand the mechanism of bone destruction in several diseases such as Rheumatoid Arthritis (RA), spondyloarthritis or osteoporosis.

RANKL is one of the most important cytokines for bone cells and for several lineages in the immune system, being essential for osteoclast differentiation/activation and for immune regulation. RANKL was originally identified as a product of $\mathrm{T}$ cells $[2,7]$, but we now know that it is also expressed by osteoblasts, osteocytes, B cells, activated T lymphocytes, synovial fibroblasts and DC. In RA, RANKL is expressed strongly in the synovium and inflammation-mediated bone damage can be largely attributed to abnormally high expression of RANKL [4,6]. By contrast, OPG is a soluble decoy receptor for RANKL that inhibits the interaction of RANKL with its specific receptor, and this represents a negative feedback loop during osteoclastogenesis [8]. In addition to its effects on osteoclast precursors, RANKL also fulfils important roles in regulating immune events, such as lymph node organogenesis and self tolerance [9].

Osteoclast precursor cells also express different kinds of receptors for proinflammatory and osteoclastogenic cytokines mainly produced by macrophages and synoviocytes, such as IL-1, TNF- $\alpha$ and IL-6. IL-1 indirectly stimulates osteoclastogenesis by acting on osteoblasts [10], whereas TNF- $\alpha$ exerts a direct effect on osteoclastogenesis by acting on osteoclast precursors, and indirectly by upregulating the production of M-CSF and RANKL by mesenchymal cells $[11,12]$. Similarly, IL-6 induces osteoclastogenesis by increasing RANKL expression in mesenchymal cells [13]. Overall, pro-inflammatory cytokines stimulate osteoclastic bone resorption in RA, both directly by acting on osteoclast precursor cells and indirectly by upregulating RANKL expression in synovial fibroblasts.

There are also several co-stimulatory molecules that act in these processes, although their role remains unclear. One of these molecules is OSCAR, an orphan IgG-like receptor that transmits an intracellular signal complementary to that of RANK, and which acts through the nuclear factor of activated T cells c1 (NFATc1) to modulate the activation and maturation of cells of the monocyte-macrophage lineage. This receptor could be relevant in the pathogenesis and severity of diseases that causes osteoclast activation. Co-stimulatory molecules use membrane adapters that contain ITAM motifs, such as DAP12 or FcR- $\gamma$. The deficiency of transmembrane signal adapters containing ITAM motifs provokes a failure in osteoclast activation and when this defect involves both molecules, it produces severe osteopetrosis [14] Interestingly, many of the transcription factors that are important for osteoclast differentiation are key regulators of immune responses, such as NF- $\mathrm{kB}$ and NFATc1. This finding was an early clue to the close relationship between skeletal biology and immunology. In summary, experimental evidence is accumulating that skeletal and immune systems share the same signalling systems and transcriptional mediators [15].

One of the main features of the rheumatoid synovium is $\mathrm{T}$ cell infiltration. When activated, T cells express RANKL, but depending on their profile they also secrete other cytokines like IFN- $\gamma$ and IL4, which have potent anti-osteoclastogenic effects even at very low concentrations. In an exhaustive study, Th1 and Th2 cells were both shown to inhibit osteoclast formation through their canonical cytokines, IFN- $\gamma$ and IL-4, respectively [16]. In contrast, Th17 cells are potent stimulators of osteoclastogenesis through the secretion of IL-17. This effect appears to be dependent on the presence of osteoblasts, leading to the conclusion that T cell-derived RANKL alone is not sufficient for osteoclast differentiation. Nowadays, there is evidence that Th17 lymphocytes play an important role not only in the inflammation but also, in the bone destruction that occurs in arthritis [16-18]. Importantly, synovial fibroblasts produce pro-inflammatory cytokines, like TNF- $\alpha$ and IL-1, promoting bone destruction by direct and indirect mechanisms. Likewise, synovial fibroblasts stimulate the migration of Th17 cells into the joint with a concomitant increase in IL17 production, promoting osteoclastogenesis through the upregulation of RANKL expression $[19,20]$.

*Corresponding author: Santos Castañeda, MD, Servicio de Reumatología, Hospital Universitario de La Princesa, c/ Diego de León 62, 28006 Madrid, Spain, Tel: +34-91-520-2200 (ext. 2473); E-mail: scastas@gmail.com

Received January 05, 2013; Accepted January 06, 2013; Published January 11 2013

Citation: Castañeda S, Arboleya L (2013) Osteoimmunology: An Evolving Discipline. J Arthritis 2: e108. doi:10.4172/2167-7921.1000e108

Copyright: (c) 2013 Castañeda S, et al. This is an open-access article distributed under the terms of the Creative Commons Attribution License, which permits unrestricted use, distribution, and reproduction in any medium, provided the original author and source are credited. 
Other important cell subsets in the field of the osteoimmunology are regulatory $\mathrm{T}$ lymphocytes (Treg lymphocytes), $\mathrm{B}$ cells and osteomas. Regulatory $\mathrm{T}$ cells play a pivotal role in the prevention of autoimmune diseases, and in vivo and in vitro data suggest that Treg cells inhibit osteoclastogenesis. However, while Treg cells can suppress osteoclastogenesis in vitro, the exact mechanism by which Treg cells affect osteoclastogenesis in physiological and pathological conditions remains to be elucidated. B cells are also a crucial element when studying immune mediated diseases and they play a pivotal role in humoral immunity. Data from animal models suggest a direct role of RANKL expression by $B$ cells in the regulation of bone metabolism [21], although the final role of B cells in promoting osteoclastogenesis is still to be fully analyzed. "Osteomacs" are another interesting cellular subset that participates in the osteoimmune network. Osteomacs are resident tissue macrophages present on the periosteal and endosteal surfaces that regulate osteoblast mineralisation, playing an intriguing role in bone homeostasis [22].

A fascinating issue that has emerged in recent years is related to bone formation. Here we must highlight the enormous advances that have occurred since the definition of the Wnt pathway. Wnt's are glycoprotein ligands that trigger multiple signal cascades involved in key processes during embryogenesis, tumourigenesis and tissue regeneration. So far, 19 different Wnt proteins have been described in mammals, acting in a variety of important signalling pathways $[23,24]$. The canonical Wnt pathway or that involving Wnt and betacatenin, is the best known signalling cascade and it plays a key role in osteoblastogenesis. There are also a number of natural antagonists of these Wnt pathways, of which sclerostin, Dickkopf (DKK) and sFRP are currently among those best known, especially DKK-1 and DKK-4 [25-27]. The activity of endogenous inhibitors of the Wnt pathway is tightly regulated due to their extraordinarily important role in many developmental processes and in the activation of several cell systems. In RA, serum levels of these inhibitors are elevated and treatment with a DKK-1 neutralizing monoclonal antibodies improves local erosions in RA hTNF. Tg mice, a model over-expressing TNF that develops an erosive arthritis resembling human RA. An exciting hypothesis would be that these inhibitors are responsible for the poor repair of erosion characteristic of RA, contrary to what occurs in other inflammatory diseases like psoriatic arthritis or ankylosing spondylitis [28].

Another interesting area of research in the field of the osteoimmunology is related to Postmenopausal (PM) osteoporosis. Oestrogen has long been known to have an impact on lymphocyte physiology and there is evidence of RANKL upregulation after ovariectomy [21]. RANKL expression in early PM women triples the number of mononuclear cells in bone marrow, both in terms of preosteoblasts or $\mathrm{T}$ and $\mathrm{B}$ lymphocytes, compared with premenopausal or PM women treated with oestrogen replacement therapy $[29,30]$. Several studies have demonstrated that oestrogen therapy modifies cytokine production by $\mathrm{T}$ cells and that of the growth factors that promote bone resorption, including the pro-osteoclastic cytokines RANKL and TNF- $\alpha[31,32]$. Thus, the physiology of T cells may be altered following oestrogen depletion, contributing to the elevated bone resorption observed in PM women [33].

Intercellular communication is another area that is providing novel and fascinating therapeutic targets. For example, Ephrin-B2 and Ephrin B4 are known to mediate the transition of bone resorption to bone formation [34]. In addition to the ephrin family and their Eph receptors, a new group of molecules has attracted great interest in recent years in the dialogue between osteoclasts and osteoblasts. These are the semaphorins, initially identified as axonal guidance molecules but subsequently involved in numerous physiological functions, including the immune and skeletal system. Indeed, osteoclast semaphorin $4 \mathrm{D}$ maintains the resorptive phase of bone remodelling by inhibiting bone formation in mice [35]. By contrast, semaphorin $3 \mathrm{~A}$ (Sema 3A) binds to neuropilin 1 (NRP-1) blocking RANKL-induced osteoclast differentiation pathways, thereby inhibiting proximal ITAM signal and RhoA while simultaneously stimulating osteoblasts through the canonical Wnt pathway. Sema3 $\mathrm{A}^{-1}$ mice develop an osteopenic phenotype that can be reproduced by shifting Sema 3A/NRP-1 signalling, while the intravenous infusion of this semaphorin increases bone volume and accelerates bone regeneration [36].

In conclusion, osteoimmunology is a new and rapidly evolving scientific discipline that involves the study of the interplay between the skeleton and the immune system, both in health and disease. Osteoimmunology has identified a wide range of molecular and cellular interactions, the detailed knowledge of which is providing a solid scientific basis for a paradigm shift in the field of inflammatory and immune diseases. The development of this discipline should enable more exciting, effective and specific therapeutic approaches to be developed for quite different diseases in a near future.

\section{Acknowledgement}

We thank Mark Sefton his help in writing and critical reading of the manuscript.

\section{References}

1. Arron JR, Choi Y (2000) Bone versus immune system. Nature 408: 535-536.

2. Wong BR, Josien R, Choi $Y$ (1999) TRANCE is a TNF family member that regulates dendritic cell and osteoclast function. J Leukoc Biol 65: 715-724

3. Fuller K, Wong B, Fox S, Choi Y, Chambers TJ (1998) TRANCE is necessary and sufficient for osteoblast-mediated activation of bone resorption in osteoclasts. J Exp Med 188: 997-1001.

4. Calvi LM, Adams GB, Weibrecht KW, Weber JM, Olson DP, et al. (2003) Osteoblastic cells regulate the haematopoietic stem cell niche. Nature 425 : 841-846.

5. Takayanagi H (2007) Osteoimmunology: shared mechanisms and crosstalk between the immune and bone systems. Nat Rev Immunol 7: 292-304.

6. Takayanagi $\mathrm{H}$ (2009) Osteoimmunology and the effects of the immune system on bone. Nat Rev Rheumatol 5: 667-676.

7. Wong BR, Josien R, Lee SY, Sauter B, Li HL, et al. (1997) TRANCE (tumo necrosis factor [TNF]-related activation-induced cytokine), a new TNF family member predominantly expressed in T cells, is a dendritic cell-specific survival factor. J Exp Med 186: 2075-2080.

8. Simonet WS, Lacey DL, Dunstan CR, Kelley M, Chang MS, et al. (1997) Osteoprotegerin: a novel secreted protein involved in the regulation of bone density. Cell 89: 309-319.

9. Leibbrandt A, Penninger JM (2010) Novel functions of RANK(L) signaling in the immune system. Adv Exp Med Biol 658: 77-94.

10. Hofbauer LC, Lacey DL, Dunstan CR, Spelsberg TC, Riggs BL, et al. (1999) Interleukin-1beta and tumor necrosis factor-alpha, but not interleukin-6, stimulate osteoprotegerin ligand gene expression in human osteoblastic cells Bone 25: 255-259.

11. Abu-Amer Y, Erdmann J, Alexopoulou L, Kollias G, Ross FP, et al. (2000) Tumor necrosis factor receptors types 1 and 2 differentially regulate osteoclastogenesis. J Biol Chem 275: 27307-27310.

12. Lam J, Takeshita S, Barker JE, Kanagawa O, Ross FP, et al. (2000) TNF-alpha induces osteoclastogenesis by direct stimulation of macrophages exposed to permissive levels of RANK ligand. J Clin Invest 106: 1481-1488.

13. Ishimi Y, Miyaura C, Jin CH, Akatsu T, Abe E, et al. (1990) IL-6 is produced by osteoblasts and induces bone resorption. J Immunol 145: 3297-3303.

14. Mocsai A, Humphrey MB, Van Ziffle JA, Hu Y, Burghardt A, et al. (2004) The 
immunomodulatory adapter proteins DAP12 and Fc receptor gamma-chain $(\mathrm{FcR}-\mathrm{y})$ regulate development of functional osteoclasts through the Syk tyrosine kinase. Proc Natl Acad Sci USA 101: 6158-6163.

15. Nakashima T, Hayashi M, Takayanagi H (2012) New insights into osteoclastogenic signaling mechanisms. Trends Endocrinol Metab 23: 582590 .

16. Sato K, Suematsu A, Okamoto K, Yamaguchi A, Morishita Y, et al. (2006) Th17 functions as an osteoclastogenic helper $T$ cell subset that links $T$ cell activation and bone destruction. J Exp Med 203: 2673-2682.

17. Annunziato F, Cosmi L, Liotta F, Maggi E, Romagnani S (2012) Defining the human T helper 17 cell phenotype. Trends Immunol 33: 505-512.

18. Komatsu N, Takayanagi $\mathrm{H}$ (2012) Inflammation and bone destruction in arthritis: synergistic activity of immune and mesenchymal cells in joints. Front Immunol 3: 77 .

19. Gravallese EM, Manning C, Tsay A, Naito A, Pan C, et al. (2000) Synovia tissue in rheumatoid arthritis is a source of osteoclast differentiation factor. Arthritis Rheum 43: 250-258.

20. Pettit AR, Walsh NC, Manning C, Goldring SR, Gravallese EM (2006) RANKL protein is expressed at the pannus-bone interface at sites of articular bone erosion in rheumatoid arthritis. Rheumatology (Oxford) 45: 1068-1076.

21. Onal M, Xiong J, Chen X, Thostenson JD, Almeida M, et al. (2012) Receptor activator of nuclear factor $\mathrm{KB}$ ligand RANKL expression by $B$ lymphocytes contributes to ovariectomy-induced bone loss. J Biol Chem 287: 29851-29860.

22. Chang MK, Raggatt LJ, Alexander KA, Kuliwaba JS, Fazzalari NL, et al. (2008) Osteal tissue macrophages are intercalated throughout human and mouse bone lining tissues and regulate osteoblast function in vitro and in vivo. $J$ Immunol 181: 1232-1244

23. Saito-Diaz K, Chen TW, Wang X, Thorne CA, Wallace HA, et al. (2013) The way Wnt works: Components and mechanism. Growth Factors 31: 1-31.

24. Clevers H, Nusse R (2012) Wnt/ $\beta$-catenin signaling and disease. Cell 149: 1192-1205.
25. Kawano Y, Kypta R (2003) Secreted antagonists of the Wnt signalling pathway. J Cell Sci 116: 2627-2634.

26. Poole KE, van Bezooijen RL, Loveridge N, Hamersma H, Papapoulos SE, et al. (2005) Sclerostin is a delayed secreted product of osteocytes that inhibits bone formation. FASEB J 19: 1842-1844.

27. Semënov M, Tamai K, He X (2005) SOST is a ligand for LRP5/LRP6 and a Wnt signaling inhibitor. J Biol Chem 280: 26770-26775.

28. Schett G, Gravallese E (2012) Bone erosion in Rheumatoid Arthritis: mechanisms, diagnosis and treatment. Nat Rev Rheumatol 8: 656-664.

29. Eghbali-Fatourechi G, Khosla S, Sanyal A, Boyle WJ, Lacey DL, et al (2003) Role of RANK ligand in mediating increased bone resorption in early postmenopausal women. J Clin Invest 111: 1221-1230.

30. Taxel P, Kaneko H, Lee S-K, Aguila HL, Raisz LG, et al. (2008) Estradiol rapidly inhibits osteoclastogenesis and RANKL expression in bone marrow cultures in postmenopausal women: a pilot study. Osteoporos Int 19: 193-199.

31. Cenci S, Weitzmann MN, Roggia C, Namba N, Novack D, et al. (2000) Estrogen deficiency induces bone loss by enhancing T-cell production of TNF-alpha. $J$ Clin Invest 106: 1229-1237.

32. D'Amelio P, Grimaldi A, Di Bella S, Brianza SZ, Cristofaro MA, et al. (2008) Estrogen deficiency increases osteoclastogenesis up-regulating $T$ cells activity: a key mechanism in osteoporosis. Bone 43: 92-100.

33. Shevde NK, Bendixen AC, Dienger KM, Pike JW (2000) Estrogens suppress RANK ligand-induced osteoclast differentiation via a stromal cell independent mechanism involving c-Jun repression. Proc Natl Acad Sci USA 97: 7829-7834.

34. Matsuo K, Irie N (2008) Osteoclast-osteoblast communication. Arch Biochem Biophys 473: 201-209.

35. Negishi-Koga T, Shinohara M, Komatsu N, Bito H, Kodama T, et al. (2011) Suppression of bone formation by osteoclastic expression of semaphorin $4 \mathrm{D}$. Nat Med 7: 1473-1480.

36. Hayashi M, Nakashima T, Taniguchi M, Kodama T, Kumanogoh A, et al. (2012) Osteoprotection by semaphorin 3A. Nature 485: 69-74. 